\title{
Implant retained nasal epithesis - case report
}

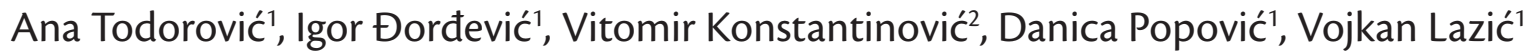 \\ 'University of Belgrade, Faculty of Dental Medicine, Department of Prosthodontics, Belgrade, Serbia; \\ ${ }^{2}$ University of Belgrade, Faculty of Dental Medicine, Department of Maxillofacial Surgery, Belgrade, Serbia
}

\begin{abstract}
SUMMARY
Nasal defects can occur as a result of head and neck trauma, or due to partial or complete ablation or resection of the tumor in the nasal area and surroundings. Smaller defects can be reconstructed surgically while large defects are mainly reconstructed combined surgically and prosthetically.

The aim of this paper was to present prosthetic reconstruction of the nasal defect by colored vinyl polysiloxane prosthesis retained with craniofacial basal disc implants.

An adequate aesthetics and stability of the prosthesis was achieved during mandible and mimic muscles movements.

Keywords: nasal defects; craniofacial disk implants; nasal prostheses; A-silicone
\end{abstract}

\section{INTRODUCTION}

Surgical defects secondary to malignant tumors (squamous cell carcinoma or basal carcinoma), and traumatic causes can result in facial disfigurement and dysfunction. Smaller facial defects can be reconstructed surgically with microvascular flaps but larger defects need to be reconstructed combined surgically and prosthetically. Poor quality or insufficient quantity of hard and soft tissue often limits treatment options. Radiation therapy especially with higher dose (70 Gy) in the treatment of malignant tumors commonly compromises bone quality and produces significant morbidity, and its consequences are unique tissue management problems $[1,2]$. Prosthetic restorations are important in the rehabilitation of such defects. However, stability and retention of nasal prosthesis can be limited due to the upper lip movement and function of mimic muscles. Retention of nasal prosthesis could be achieved through eyeglasses, skin adhesives or craniofacial implants [1].

In 1977, Branemark et al. introduced the concept of endosseous implants as anchorage for facial prosthetic devices [3]. This concept was upgraded until today and used to retain almost all facial prosthesis. These extraoral implants are shorter than the ones used intraorally, which, therefore, allow their placement in pericranial bone $[3,4,5]$. Rehabilitation of patients with bone-anchored prosthesis has several advantages over conventional methods, where use of skin adhesives is necessary for retention. Adhesives can cause skin irritation, allergic reaction, discoloration of prosthetic material, and accidental detachment $[6,7]$. Another option for extraoral implantology is the use of disc implants (Basal Osseointegration Implants) via basal osseointegration in compact bone that is resistant to resorption. Advantages of these implants include cortical anchorage, ability to add more bone screws for stabilization, and modest demand for vertical (axial) bone. In the midface area, anchorage of screw-type implants is difficult because axial bone supply is limited and only thin plates of cortical bone are present. The only locations for screw implant placement are glabella and upper (basal) alveolar crest of maxilla. If vertical or even horizontal bone dimensions are limited in the alveolar crest due to atrophy or after resection, axial implants often cannot be used [8]. The most commonly used material for producing facial prosthesis is colored methyl methacrylics or RTV (Room Vulcanisation Silicone) vinyl polysiloxane colored silicone [1].

The aim of this article was to present a clinical report of a patient who underwent facial reconstruction with nasal epithesis anchored on disk implants after ablation of midface due to the present squamous cell carcinoma.

\section{CLINICAL CASE}

A 65-year-old woman with a large nasal tumor was referred to the Clinic for Maxillofacial surgery at the Faculty of Dental Medicine, University of Belgrade. The patient stated that first observation of the slow-growing tumor happened a year before the admission for surgery. Histopathological diagnosis of squamous cell carcinoma was obtained after an incisional biopsy. When nasal pyramid was amputated and tumor entirely removed due to limited bone substance basal (disk) implants were indicated as the optimal solution. In surgical stage, basal implants (Diskos; Dr. Ihde Dental AG, Switzerland) were inserted for anchorage of the nasal prosthesis in the nasal floor and glabellar region (Figures 1,2). The main advantage of basal (disk) implants is relatively small diameter of the vertical part $(2.3 \mathrm{~mm})$ and bicortical implantation technique for achieving excellent primary implant stabilization. After an unloaded osseointegration phase of 3 


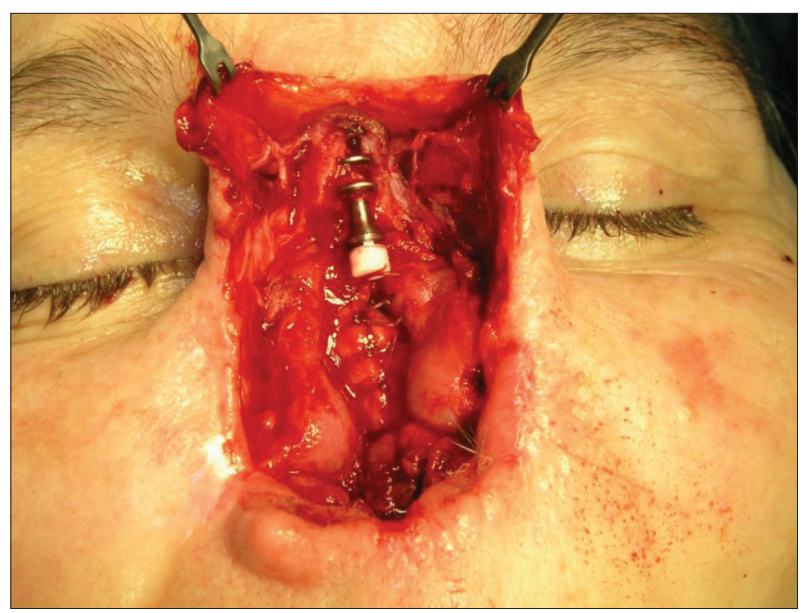

Figure 1. Surgical insertion of $\mathrm{BOI}$ implant in glabela region Slika 1. Hirurška ugradnja bazalnog disk implantata u predelu glabele

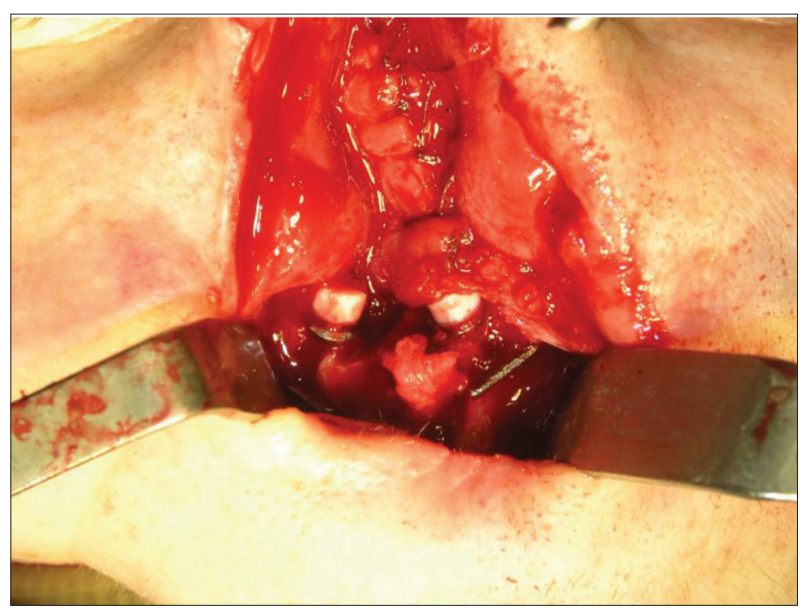

Figure 2. Surgical insertion of two BOI implanst in the nasal floor Slika 2. Hirurška ugradnja dva bazalna disk implantata u predelu poda nosa

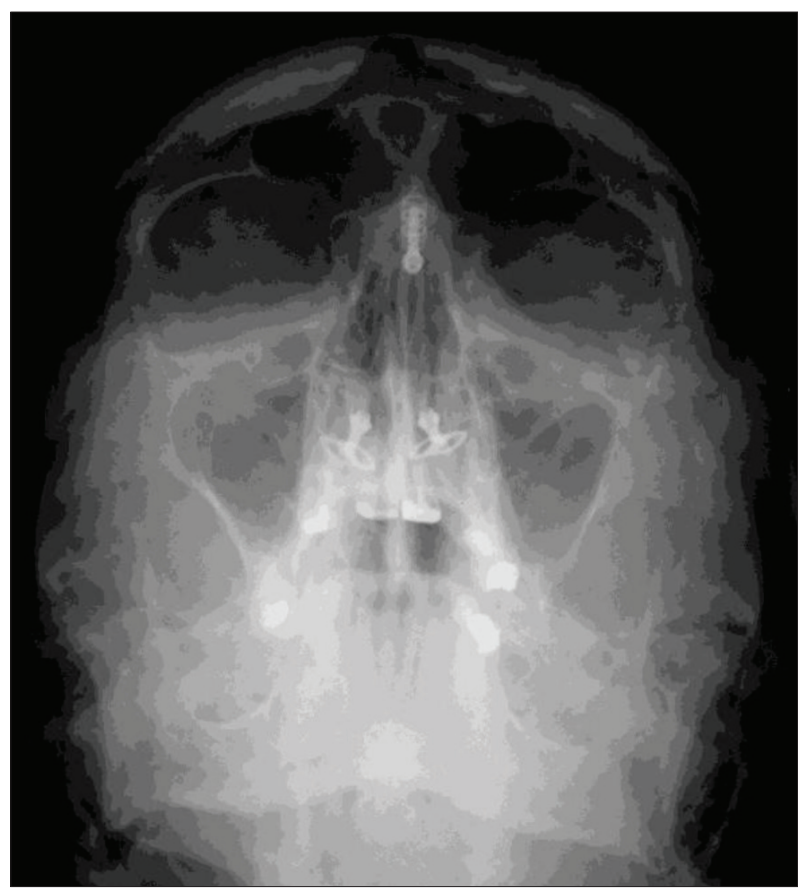

Figure 3. Control $\mathrm{X}$ ray after period of osseointegration of $\mathrm{BOI}$ disc implants

Slika 3. Kontrolni RDG snimak oseointegracije implantata

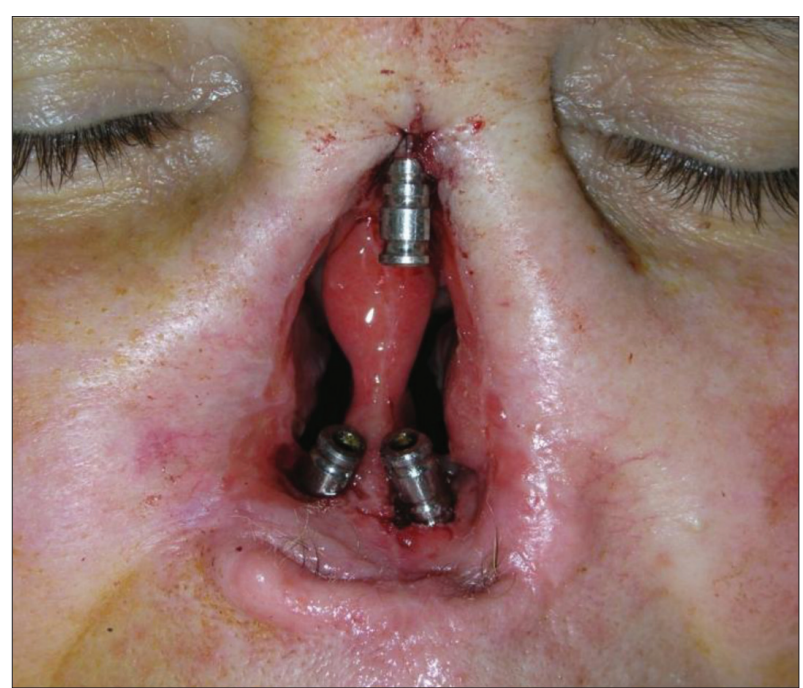

Figure 4. Inserted implant transfers on $\mathrm{BO}$ disc implants Slika 4. Postavljeni transferi disk implantata BOI

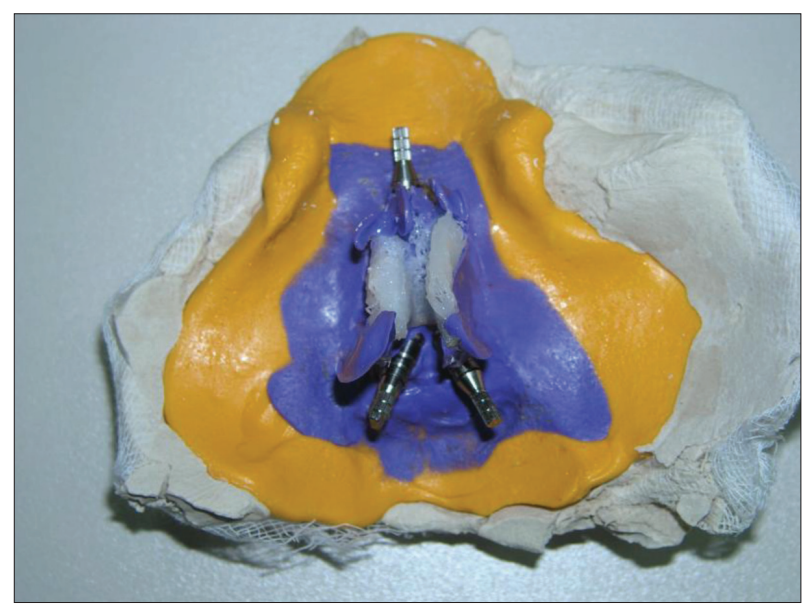

Figure 5. VPS impression with inserted implant analogues Slika 5. Otisak VPS otisnim materijalima sa postavljenim analozima implantata

months, all implants appeared well integrated according to the radiologic criteria and clinical stability (Figure 3). At the end of the osseointegration process, the final nasal prosthesis was produced. Before taking the impression of the defect implant position transfers were placed and implant level impression taken with A-silicones (Figures $4,5)$. Epithesis was anchored through Hader bars that were fabricated to provide better retention and stability of the prosthesis (Figure 6). Hader bars were casted from chromium-cobalt alloy through prefabricated plastic models (CEKA, Netherlands). Acrylic resin substructures housing with the plastic retentive clips were designed and fabricated for better support of silicone prosthesis (Figure 7). The wax sculpting of the prosthesis was prepared and evaluated on the patient, ensuring that the pattern truly restored contour and symmetry (Figure 8). Nasal prosthesis was made from A-silicone material with intrinsic and extrinsic coloration procedures (Epithetik Set; Bredent, Germany). Plastic clips on the acrylic resin substructure bars screwed on the double-plate disc implants retained the silicon nasal prosthesis. The prosthesis was finally delivered to the patient (Figure 9) and hygiene instructions provided in the usual manner. After 3, 6 and 12 months, 


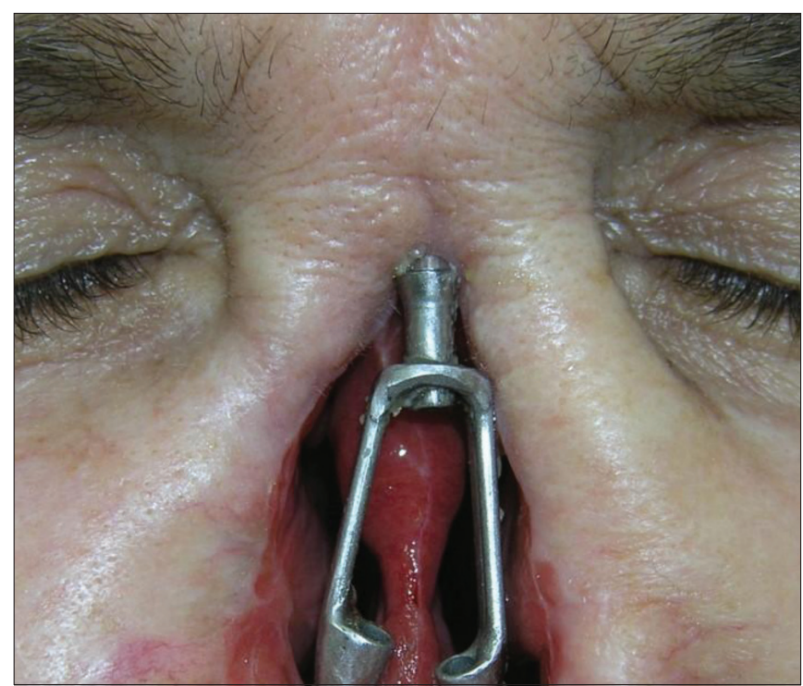

Figure 6. Hader bar on BOI implants

Slika 6. Postavljena prečka Hader na implantate BOI

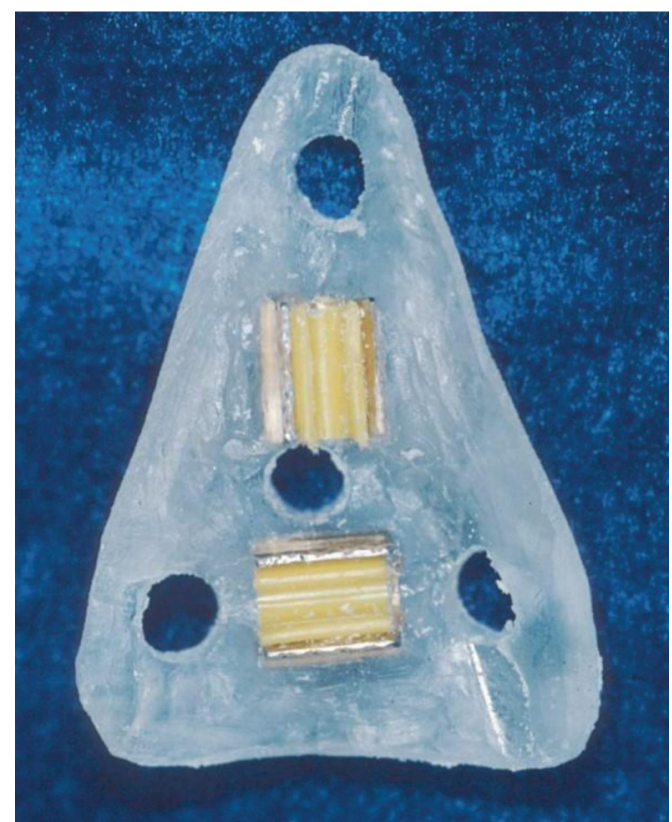

Figure 7. Acrylic base of silicone nasal prosthesis with matrices Slika 7. Akrilatna baza proteze nosa od silikona sa matricama

on the control visits, the patient was satisfied with aesthetic and functional characteristics of the prosthesis.

\section{DISCUSSION}

Facial prostheses can efficiently restore relatively fixed facial structures such as auricular, nasal, or upper lip prostheses. Reconstructed cheek defect by free flaps provides support, stability and retention of maxillary obturator prosthesis while obturator prosthesis on the other hand provide cheek support and allow symmetry of the middle face.

In our case, a decision was made for surgical - prosthetic treatment of nasal defect formed after ablation of complete nose pyramid due to malignant tumor. Reconstruction of large defect of the middle face was not possible with microvascular surgery procedures. The patient

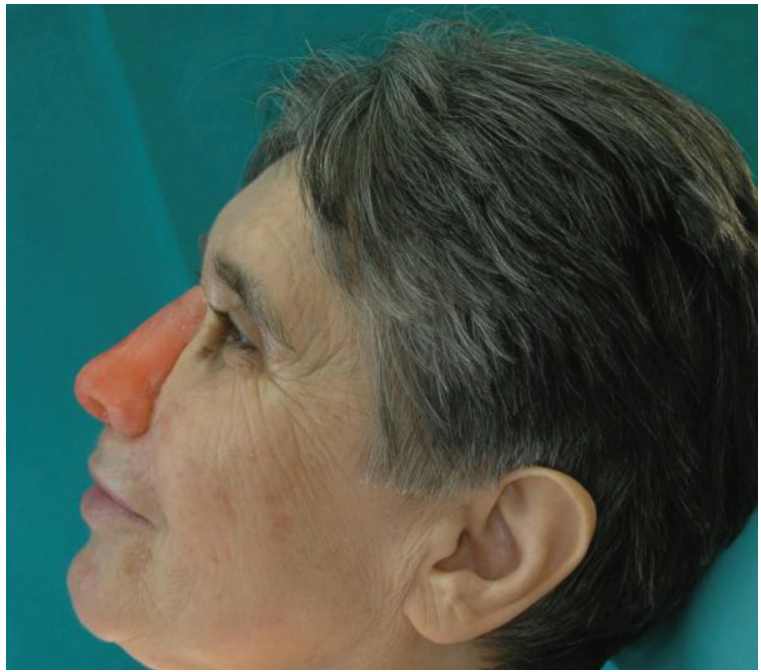

Figure 8. Try in of wax sculpted nasal prosthesis Slika 8. Voštani model proteze nosa

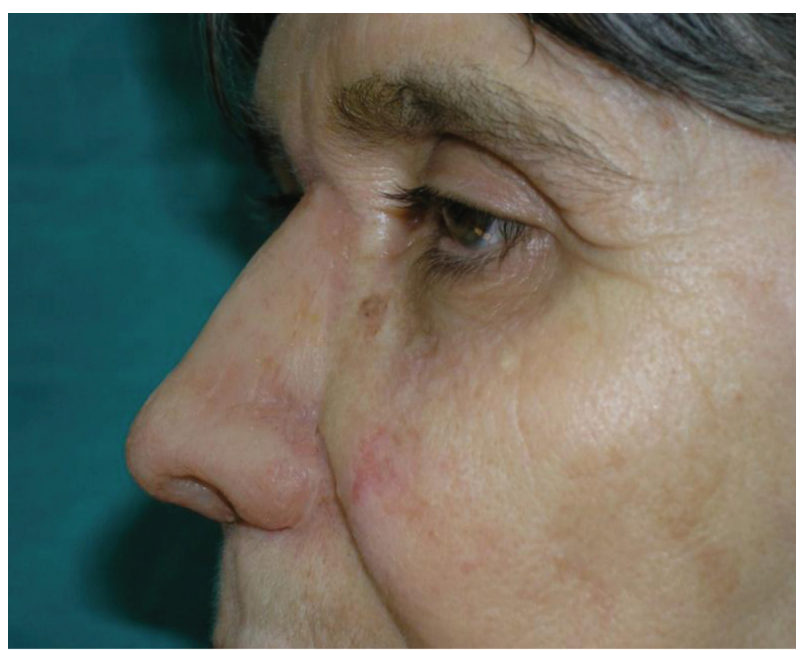

Figure 9. Implant retained silicone nasal prosthesis Slika 9. Implantatno retinirana proteza nosa od silikona

was presented with a need for implantation of craniofacial implants in the area of glabella and the floor of the nose so that the prosthesis would optimally be retained and stabilized and then produced from RTV silicone with a thin acrylic base as suggested by Roumanas et al. [9]. That kind of prosthesis would be lighter, more aesthetic and it would not move much during oral functions $[1,2,6,7,9]$.

Several factors should be considered when designing a retention system for facial prosthesis to avoid implant failure or complications. It is desirable to connect all implants with a rigid bar. Consequently, the stress delivered to the implants would be distributed equally among the implants. Also, the retention bars must fit in passive manner and retention system must fit within the confines of the prosthesis without affecting contour or symmetry. Retention must be sufficient to eliminate accidental dislodgement. Basal implants use cortical bone for implant anchorage. The void spaces created by the osteotomy fill with blood, which is later reorganized to become woven bone and, finally, osteonal bone. Thus, basal implants exhibit a dual integration process similar to orthopedic healing patterns after long bone fractures. 


\section{CONCLUSION}

Excellent stability, retention and outstanding function and aesthetics can be achieved with surgical and prosthetic procedures in the reconstruction of middle part of the face defects by the implant retained silicone nose prosthesis. This treatment is very important for socialization of patients with visible facial defects.

\section{REFERENCES}

1. Beumer J, Curtis TA, Marunick MT. Maxillofacial Rehabilitation, Prosthodontic and Surgical Considerations. St Louis: Ishiyaku EuroAmerica. 1996; 377-453.

2. Padmanabhan TV, Mohamed K, Parameswari D, Nitin SK. Prosthetic rehabilitation of an orbital and facial defect: A Clinical Report. J Prosthodont. 2012; 21(3):200-4. [DOI: 10.1111/j.1532849X.2011.00817.x] [PMID: 22356269]

3. Branemark PI, Albrektsson T. Titanium implants permanently penetrating human skin. Scand I Plast Reconstr Surg. 1982; 16(1):17-21. [PMID: 7112035]
4. Tjellstrom A, Jacobsson M, Albrektsson T, Jannson K. Use of tissue integrated implants in congenital aural malformations. Adv Otorhinolaryngol. 1988; 40:24-32. [PMID: 3389230]

5. Tjellstrom A, Yontchev E, Lindstrom J, Branemark PI. Five years' experience with bone-anchored auricular prostheses. Otolaryngol Head Neck Surg. 1985; 93(3):366-72. [DOl: 10.1177/019459988509300315] [PMID: 3927232]

6. Cantor R, Hildestad P. A material for epitheses. A preliminary report. Odontol Tidskr. 1966; 74(1):32-40. [PMID: 5326048]

7. Chang TL, Garrett N, Roumanas E, Beumer J 3rd. Treatment satisfaction with facial prostheses. J Prosthet Dent. 2005; 94(3):275-80. [DOI: 10.1016/j.prosdent.2005.06.002] [PMID: 16126080]

8. Konstantinović VS, Lazić VM, Stefan I. The Nasal Epithesis Retained by Basal (Disk) Implants. J Craniofac Surg. 2010; 21(1):33-6. [DOI: 10.1097/SCS.0b013e3181c3626c] [PMID: 20061980]

9. Roumanas ED, Chang TL, Beumer J. Use of osseointegrated implants in the restoration of head and neck defects. J Calif Dent Assoc. 2006; 34(9):711-8. [PMID: 170222295]

Received: 09.01.2017• Accepted: 18.05.2017 


\title{
Retencija proteze nosa pomoću implantata - prikaz slučaja
}

\author{
Ana Todorović 1 , Igor Đorđević 1 , Vitomir Konstantinović ${ }^{2}$, Danica Popović1, Vojkan Lazić \\ 'Univerzitet u Beogradu, Stomatološki fakultet, Klinika za stomatološku protetiku, Beograd, Srbija; \\ ${ }^{2}$ Univerzitet u Beogradu, Stomatološki fakultet, Klinika za maksilofacijalnu hirurgiju, Beograd, Srbija
}

\begin{abstract}
KRATAK SADRŽAJ
Defekti nosa mogu nastati kao posledica traume glave i vrata ili usled delimične ili potpune ablacije, odnosno resekcije tumora predela nosa i okoline. Manji defekti nosa se rekonstruišu hirurški, dok se veliki defekti uglavnom rekonstruišu kombinovano, kako hirurški tako i protetski.

Cilj ovoga rada bio je da se prikaže protetska rekonstrukcija defekta nosa protezom izrađenom od vinil-polisiloksana u boji sa retencijom preko kraniofacijalnih bazalnih disk-implantata.

Ovakvom protetskom nadoknadom postignute su zadovoljavajuća estetika i stabilnost proteze pri pokretima mandibule i mimičnih mišića.

Ključne reči: defekt nosa; kraniofacijalni disk-implantati; proteza nosa; A-silikon
\end{abstract}

\section{UVOD}

Defekti nosa najčešće nastaju usled kompletne ili delimične ablacije (resekcije) tumora (planocelularni i bazocelularni karcinomi) ili kao posledica traume predela glave i vrata. Manji defekti se uglavnom rekonstruišu hirurški, putem režnjeva sa vaskularnom peteljkom, dok se veliki defekti rekonstruišu hirurški i protetski - protezama nosa. Hirurška rekonstrukcija može biti ograničena stanjem i količinom preostalog koštanog i mekog tkiva lica, odnosno velikom dozom zračenja regiona (70 Gy) kod malignih tumora i značajnim stepenom oštećenja lokalne vaskularne mreže kao posledice zračne terapije [1,2]. Protetska rekonstrukcija defekata nosa je jedino moguće rešenje, ali može biti limitirana pokretima gornje usne, kao i funkcijom mimičnih mišića, to jest problemom retencije proteza za lice pacijenata. Retencija se može obezbediti preko okvira naočara kod proteza izrađenih od metil-metakrilata, dok se proteze lica od silikona mogu pričvrstiti ili pomoću posebnog lepka za kožu ili putem kraniofacijalnih implantata [1].

Branemark i saradnici su 1977. godine [3] predstavili koncept hirurške ugradnje implantata manjih dimenzija radi retencije aurikularne proteze. Međutim, taj koncept je unapređivan i danas se koristi veoma često prilikom hirurško-protetske rekonstrukcije defekata lica različitim nadoknadama. Ovi ekstraoralni implantati su kraći nego intraoralni i zato ih je moguće ugraditi u perikranijalnu kost $[3,4,5]$.

Rehabilitacija pacijenata implantatno-nošenim protezama ima nekoliko prednosti u odnosu na kovencionalni metod, gde je neophodna upotreba adheziva za kožu radi retencije. Adhezivi mogu izazvati iritaciju kože, alergijske reakcije, diskoloraciju protetskog materijala i odvajanje proteze od kože $[6,7]$.

Drugu mogućnost u ekstaoralnoj implantologiji predstavlja upotreba disk-implantata (Basal Osseointegration Implants) putem bazalne oseointegracije u kompaktnu kost, koja je otporna na resorpciju. Prednosti pri postavljanju ovih implantata uključuju kortikalno sidrenje, mogućnost dodavanja više koštanih šrafova za stabilizaciju i skromne zahteve za prisustvo adekvatne vertikalne (aksijalne) dimenzije kosti. U srednjoj regiji lica sidrenje implantata tipa šrafa je otežano, jer je ograničena raspoloživost vertikalne dimenzije kosti i prisutne su samo tanke ploče kortikalne kosti. Jedine povoljne lokacije za postavljanje ekstraoralnih implantata su glabela i gornji (bazalni) alveolarni nastavak maksile. Ako su na alveolarnom grebenu ograničene vertikalna ili čak i horizontalna dimenzija kosti zbog atrofije ili nakon resekcije, aksijalni implantati se često ne mogu upotrebiti [8].

Materijal od kojeg se najčešće izrađuju proteze lica je metilmetakrilat u boji ili RTV (Room Temperature Vulcanization Silicone) vinil-polisiloksan (silikon) u boji [1]

Cilj ovoga rada bio je da se na jednom slučaju iz kliničke prakse prikažu protetske rekonstrukcije nosa pomoću implantatno retinirane proteze nakon hirurške ablacije planocelularnog karcinoma srednjeg dela lica.

\section{PRIKAZ SLUČAJA}

Pacijentkinja 65 godina starosti, sa tumorom nosa, javila se na pregled na Kliniku za maksilofacijalnu hirurgiju Stomatološkog fakulteta Univerziteta u Beogradu. Pacijentkinja je izjavila da je prvi put primetila spororastući tumor godinu dana pre prijema na hirurgiju.

Histopatološka dijagnoza skvamocelularnog karcinoma je dobijena nakon incizione biopsije. Kada je amputirana nazalna piramida i tumor potpuno ukljonjen, zbog male preostale koštane supstance indikovani su bazalni (disk) implantati kao optimalno rešenje. U istoj hirurškoj fazi bazalni implantati (Diskos; Dr. Ihde Dental AG, Switzerland) postavljeni su kao mesto sidrenja nazalne proteze na podu nosa i glabelarnom predelu (Slike $1,2)$. Glavne prednosti bazalnih (disk) implantata su relativno mali dijametar vertikalnog dela $(2,3 \mathrm{~mm})$ i bikortikalna tehnika ugradnje, za postizanje odlične primarne implantatne stabilnosti. Posle perioda oseointegracije od tri meseca, svi implantati su bili dobro integrisani na osnovu radioloških kriterijuma i kliničke stabilnosti (Slika 3). Na kraju procesa oseointegracije izrađena je definitivna nazalna proteza. Pre uzimanja otiska defekta postavljeni su prenosnici položaja implantata i realizovan je otisak na nivou implantata adicionim silikonom (Slike 4,5). Epiteza je usidrena pomoću prečki Hader, koje su proizvedene da obezbede bolju retenciju i stabilnost proteze (Slika 6). Prečka Hader je izlivena od legure kobalt-hrom pomoću prefabrikovanih plastičnih modela (CEKA, Netherlands). Akrilatno kućište sa plastičnim matricama za retenciju je dizajnirano i proizvedeno za bolju potporu silikonske proteze (Slika 7). Modelacija proteze u vosku je 
pripremljena i evaluirana na pacijentu, kako bi se obezbedilo da model verno restauriše konturu i simetriju (Slika 8). Nazalna proteza izrađena je od adicionog silikona uz korišćenje intrinzičkih i ekstrinzičkih procedura bojenja (Epithetik Set; Bredent, Germany). Nazalna proteza od silikona je retinirana plastičnim matricama na akrilnoj substrukturi prečki ušrafljenih na diskimplantatima. Proteza je predata pacijentkinji (Slika 9) i data su joj uputstva za održavanje higijene. Nakon 3, 6 i 12 meseci, na kontrolnim pregledima, pacijentkinja je bila zadovoljna i estetskim i funkcionalnim karakteristikama proteze.

\section{DISKUSIJA}

Protezama lica se veoma efikasno restauriraju strukture koje su relativno pokretne, kao što su aurikularne, nazalne ili proteze koje nadoknađuju gornju usnu. Obraz rekonstruisan slobodnim mikrovaskularnim režnjem pruža potporu, stabilnost i retenciju maksilarne opturator proteze, dok opturacioni segment proteze, s druge strane, takođe pruža potporu rekonstruisanom obrazu i omogućuje simetričnost srednjeg facijalnog masiva. U ovom slučaju doneta je odluka za hirurško-protetsku terapiju defekta nosa nastalog posle ablacije kompletne piramide nosa sa malignim tumorom. Rekonstrukcija velikog defekta srednjeg masiva lica nije bila moguća postupcima mikrovaskularne hirurgije. Pacijentu je predočena potreba ugradnje kraniofacijalnih implantata u predelu glabele i poda nosa kako bi se proteza nosa optimalnije retinirala i stabilizovala, a tada bi mogla da se izradi od RTV silikona sa tankom akrilatnom bazom, kako se navodi u istraživanju Roumanasa i saradnika [9]. Takva proteza bi bila lakša i ne bi se mnogo pomerala u toku oralnih funkcija, a postigla bi se i visoka estetika $[1,2,6,7,9]$. Nekoliko faktora treba uzeti u obzir pri dizajniranju retencionog sistema proteza u predelu lica nošenim implantatima kako bi se izbegle moguće komplikacije gubitka implantata [1]. Neophodno je povezati sve implantate pomoću rigidne konekcije tipa prečke. Posledično, stres koji prihvataju implantati će se distribuirati podjednako između implantata. Retenciona prečka mora nalegati pasivno na implantate, a retencioni sistem se mora ukopiti u okviru granica proteze bez uticaja na njene konture ili simetriju. Retencija mora biti odgovarajuća kako bi se sprečilo akcidentalno odvajanje proteze. Bazalni implantati koriste kortikalnu kost za sidrenje implantata. Prazni prostori koji nastaju posle osteotomije se pune krvlju, što kasnije postaje nezrela vlaknasta kost i konačno zrela lamelarna kost. Na taj način bazalni implantati pokazuju dvostruki proces integracije, koji je sličan ortopedskom zarastanju nakon fraktura dugih kostiju.

\section{ZAKLJUČAK}

Hirurško-protetskim postupcima rekonstrukcije defekata srednjeg masiva lica putem implantatno retiniranih proteza nosa od silikona mogu se postići odlična stabilnost, retencija, kao i izvanredna funkcija i estetika, koja je važna za socijalizaciju pacijenata sa vidljivim defektima lica. 